PREPARED FOR THE U.S. DEPARTMENT OF ENERGY, UNDER CONTRACT DE-AC02-76CH03073

PPPL-4004

PPPL-4004

UC-70

Fusion Reaction Rate

in an Inhomogeneous Plasma

by

S. Son and N.J. Fisch

September 2004

N/W

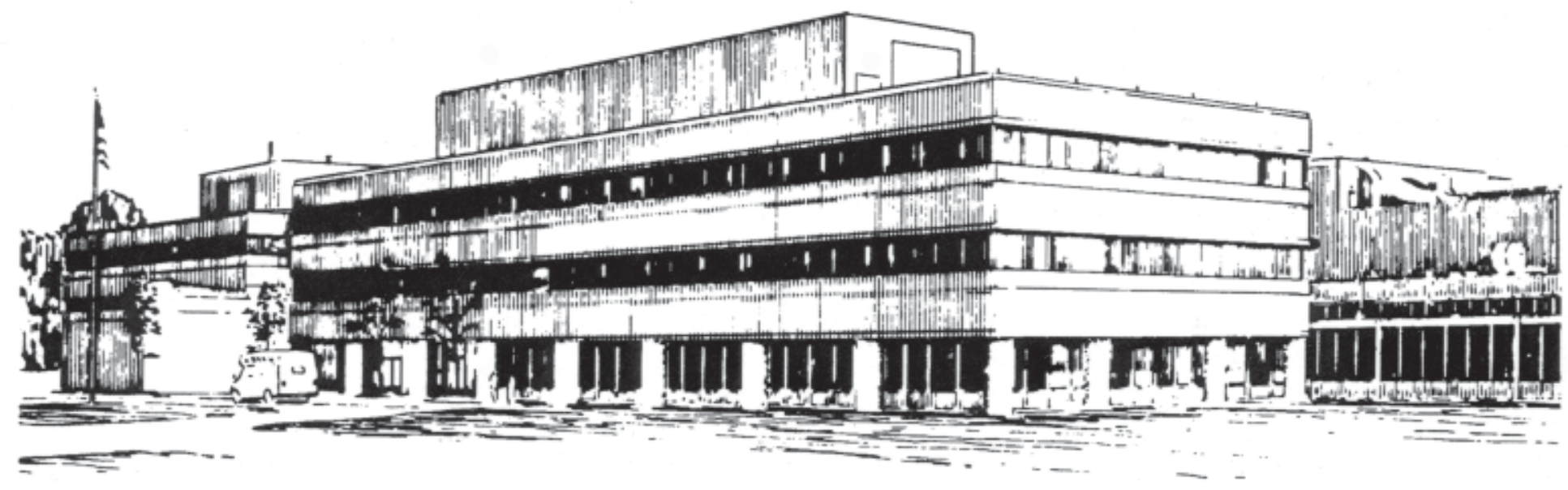

PRINCETON PLASMA PHYSICS LABORATORY PRINCETON UNIVERSITY, PRINCETON, NEW JERSEY 


\section{PPPL Reports Disclaimer}

This report was prepared as an account of work sponsored by an agency of the United States Government. Neither the United States Government nor any agency thereof, nor any of their employees, makes any warranty, express or implied, or assumes any legal liability or responsibility for the accuracy, completeness, or usefulness of any information, apparatus, product, or process disclosed, or represents that its use would not infringe privately owned rights. Reference herein to any specific commercial product, process, or service by trade name, trademark, manufacturer, or otherwise, does not necessarily constitute or imply its endorsement, recommendation, or favoring by the United States Government or any agency thereof. The views and opinions of authors expressed herein do not necessarily state or reflect those of the United States Government or any agency thereof.

\section{Availability}

This report is posted on the U.S. Department of Energy's Princeton Plasma Physics Laboratory Publications and Reports web site in Fiscal Year 2004. The home page for PPPL Reports and Publications is: http://www.pppl.gov/pub_report/

DOE and DOE Contractors can obtain copies of this report from:

U.S. Department of Energy

Office of Scientific and Technical Information

DOE Technical Information Services (DTIS)

P.O. Box 62

Oak Ridge, TN 37831

Telephone: (865) 576-8401

Fax: (865) 576-5728

Email: reports@adonis.osti.gov

This report is available to the general public from:

National Technical Information Service

U.S. Department of Commerce

5285 Port Royal Road

Springfield, VA 22161

Telephone: $1-800-553-6847$ or

(703) $605-6000$

Fax: (703) 321-8547

Internet: http://www.ntis.gov/ordering.htm 


\title{
Fusion Reaction Rate in an Inhomogeneous Plasma
}

\author{
S.Son* and N.J. Fisch ${ }^{\dagger}$ \\ Princeton Plasma Physics Laboratory
}

\begin{abstract}
The local fusion rate, obtained from the assumption that the distribution is a local Maxwellian, is inaccurate if mean-free-paths of fusing particles are not sufficiently small compared with the inhomogeneity length of the plasma. We calculate the first order correction of $P_{0}$ in terms of the small spatial gradient and obtain a non-local modification of $P_{0}$ in a shock region when the gradient is not small. Use is made of the fact that the fusion reaction cross-section has a relatively sharp peak as a function of energy.
\end{abstract}

PACS numbers: 51.10.+y

${ }^{*}$ Electronic address: sson@pppl.gov

${ }^{\dagger}$ Electronic address: fisch@pppl.gov 


\section{INTRODUCTION}

Transport coefficients are derived using the deviation of particle distributions from a local Maxwellian through the so-called "Chapman-Enskog-Procedure" (CEP) [1-7]. CEP correctly predicts the distribution $f(E)$ when the spatial scale $L$ of the plasma is larger than the mean-free-path $\lambda_{E}$ of an ion with energy $E$ ( when $\left.\lambda_{E} \ll L\right)$.

For Coulomb collisions, the length $\lambda_{E}$ is proportional to $E^{3 / 2}$, so the assumption $\lambda_{E} \ll L$ does not hold for high energy $E$. When $\lambda_{E} \cong L$, the error permitted by CEP is as large as the value predicted:

$$
\left|\frac{f_{C}(E)-f(E)}{f(E)}\right| \cong 1
$$

where $f_{C}$ is first-order corrected distribution from CEP and $f$ is the exact distribution [5]. CEP then fails to predict the distirbution function at high energy. It is even true that $\left|\left(f_{C}(E)-f(E)\right) / f(E)\right| \rightarrow \infty$ as $E \rightarrow \infty$.

For convenience, let us define the "breakdown region" as the phase space where CEP fails ( as in Eq.(1) ). We define also a "breakdown particles" as the particles in that region. If the breakdown region contains the phase space in which the occurrence of a physical process is sensitive, such as the high energy limit above, CEP is not accurate to use for physical quantities related to the process. Examples might include the electric heat conductivity, the nuclear reaction rate and the ionization rate, all of which are sensitive to energetic particles.

In the case of the electric heat conductivity, electrons responsible for the heat transfer are those with kinetic energies higher than the temperature. If their mean-free-paths are larger than or comparable to the gradient scale length, CEP overestimates the actual heat conductivity [6, 8-17]. In the case of the nuclear reaction rate, fusing ions have much higher energies than the average ions [18]. If their mean-free-paths are not small compared to the inhomogeneity length of a plasma, the fusion rate formula, $P_{0}(n, T)$, from a local Maxwellian assumption is only approximate.

In this paper, we consider the reaction rate change due to the particle-distribution deviation from a local Maxwellian in the presence of a spatial inhomogeneity. We identify a region for which CEP is applicable. When CEP is valid, we calculate a modified form of the fusion rate from $P_{0}(n, T)$ to $P_{0}(n, T)+\delta P(n, \nabla n, T, \nabla T, V, \nabla V)$. When CEP is not valid, we obtain a considerably different rate because of the non-local effects in a shock region. 
The paper is organized as follows. In Sec.II, basic physics are introduced for the fusion rate and CEP. In Sec.III, $P_{0}(n, T)+\delta P(n, \nabla n, T, \nabla T, V, \nabla V)$ is calculated when the spatial length scale is large. In Sec.IV, the shock region is studied as a special case when CEP breaks down. In Sec.V and VI, a discussion and a conclusion are given.

\section{BASIC EQUATION}

\section{A. Nuclear Reaction Rate}

The reaction rate $P$ in a plasma is determined by the distribution function $f$ and the cross-section $\sigma$ :

$$
P=\int f_{1}\left(v_{1}\right) f_{2}\left(v_{2}\right) \sigma\left(\left|v_{1}-v_{2}\right|\right)\left|v_{1}-v_{2}\right| d v_{1} d v_{2}
$$

where $P$ is in units of $\mathrm{cm}^{-3} \mathrm{sec}^{-1}$, or events per unit time per unit volume. The cross-section is proportional to the contact probability of two particles. It is virtually zero under classical mechanics, and the barrier penetration is possible only through quantum effects. Two-body quantum mechanics gives the contact probability, which is proportional to the amplitude of the wave function at zero distance of fusing pair [18, 19]. The cross-section is given [18] as

$$
\sigma_{i, j}(E)=\frac{S_{i, j}(E)}{E} e^{-\pi\left(\frac{E_{G}}{E}\right)^{1 / 2}}
$$

where $E_{G}=Z_{i} Z_{j} e^{2} / r_{i, j}=50\left(Z_{i} Z_{j}\right)^{2}\left(2 \mu_{i j} / m_{N}\right) \mathrm{keV}, r_{i, j}=\hbar^{2} / 2 \mu Z_{i} Z_{j} e^{2}, u_{i, j}$ is the relative mass, $E$ is the relative kinetic energy, $S_{i, j}$ is a slowly varying function of energy, and $m_{N}$ is in esu.

If the distribution function is a Maxwellian, the fusion rate can be written a function of density $\mathrm{n}$ and temperature $\mathrm{T}$ only. Since a Maxwellian distribution decreases with energy, and the cross-section increases with energy, there exists a maximum of the integrand in Eq.(2) responsible for most of the reactions. The energy at the maximum, the so-called " Gamow Peak", is

$$
E_{G p}=\frac{1}{3} \tau_{i j} k_{b} T
$$


where $\tau_{i j}=3(\pi / 2)^{2 / 3}\left(E_{G} / k_{B} T\right)^{1 / 3}$. The peak is much larger than the temperature of the plasma in most cases [18]. The method of the steepest-descents can be used for the integration in Eq.(2), giving the local fusion rate [18]

$$
P_{0}(n, T)\left(\frac{1}{\mathrm{~cm}^{3} \mathrm{sec}}\right)=n_{i} n_{j} \tau_{i j}^{2} \exp \left(-\tau_{i j}\right)\left[\frac{16 S_{i, j}(T) r_{i, j}}{3^{5 / 2} \pi \hbar}\right]
$$

\section{B. Chapman Enskog Procedure}

We first summarize the Chapman-Enskog procedure [4]. To calculate transport coefficients, we solve the Vlasov equation with a collisional model:

$$
\frac{D f}{D t}=\frac{\partial f}{\partial t}+\mathbf{v} \cdot \nabla f+\frac{q}{m} \mathbf{E} \cdot \nabla_{v} f=-C(f) .
$$

If macroscopic quantities, such as the density and temperature, vary slowly compared to the collision frequency, a perturbation expansion is possible [1-5]. To zeroth order, the right hand side of Eq.(6) is zero, since the collision frequency is the fastest time scale. The

zeroth order solution is then local Maxwellian $f_{0}=n(x) f_{M}\left(\frac{m(v-u(x))^{2}}{2 T(x)}\right)$. Note $C\left(f_{0}\right)=0$, but $\frac{D f_{0}}{D t} \neq 0$, due to the parametric dependence. The next order equation will be

$$
\frac{D f_{0}}{D t}=-C\left(f_{1}\right)
$$

An Euler equation will result if we take the conserved moments of Eq.(7), thereby imposing constraints on the density, the temperature and the velocity, i.e.

$$
\left(\begin{array}{c}
\frac{\partial n}{\partial t}+\nabla \cdot(n \mathbf{u})=0 \\
\rho_{m} \frac{d \mathbf{u}}{d t}=n q \mathbf{E}-\nabla \cdot(n T \hat{I}) \\
\frac{3}{2} n \frac{d T}{d t}=-(\nabla \mathbf{u}):(n T \hat{I})
\end{array}\right)
$$

where $\hat{I}$ is the identity matrix and $A: B=\Sigma_{i, j} A_{i, j} B_{i, j}$. By using the above equations, $\frac{D f_{0}}{D t}$ can be simplified to

$$
\left.\frac{D f_{0}}{D t}=f_{M}\left[\left(\frac{1}{2} \frac{\delta v^{2}}{v_{t}^{2}}-\frac{5}{2}\right) \delta \mathbf{v} \cdot \nabla \ln T+\frac{1}{v_{t}^{2}}\left(\delta v \delta v-\frac{1}{3} \delta v^{2} \hat{I}\right): \hat{S}\right)\right]
$$


where $v_{t}=\sqrt{T / m}$, and $\hat{S}_{i, j}=\frac{1}{2}\left(\frac{\partial u_{i}}{\partial x_{j}}+\frac{\partial u_{j}}{\partial x_{i}}\right)$. By solving Eq.(7) with Eq.(8) for the ions, we obtain $f_{1}$ and the rate from Eq.(2).

For the collision operator, we use the Landau operator:

$$
C_{r, s}=A(r, s) \Sigma_{k, p}\left(\frac{\partial}{\partial v}\right)_{k} \cdot\left[\int \hat{U}_{k, p}\left(\vec{v}-\overrightarrow{v^{\prime}}\right) \cdot\left[\frac{1}{m_{s}}\left(\frac{1}{\partial v_{s}^{\prime}}\right)_{p}-\frac{1}{m_{r}}\left(\frac{1}{\partial v_{r}}\right)_{p}\right] f_{r}(v) f_{s}\left(v^{\prime}\right)\right] d^{3} v^{\prime},
$$

where $A_{r, s}=2 \pi\left(q_{r}^{2} / m_{r}\right)\left(n_{s} q_{s}^{2}\right) \ln \Lambda$ and $\hat{U}_{k, p}(\vec{v})=\frac{v^{2} \delta k, p-v_{k} v_{p}}{|v|^{3}}$ is a tensor. We now compute $C_{i, e}$ and $C_{i, j}$. We linearize $C_{i, j}$ and $C_{i, e}$ around a local Maxwellian using $f_{i}=f_{M}(1+\chi)$. Fusing particles are much faster than average particles from Eq.(5), and they collide most often with average particles, so we use $v_{i} \gg v_{j}$ in computing $C_{i, j}\left(\chi_{i}\right)$. Thermal electrons are much faster than the fusing particles, so we use $v_{e} \gg v_{i}$ in computing $C_{i, e}$. The linearized collision operator obtained from these assumptions is given as

$$
\left(\begin{array}{c}
C_{i, j}(\chi)=-A(i, j)\left[\frac{f_{M}}{m_{i} v^{3}}\left(L^{2} \chi-2 v \frac{m_{i}}{m_{j}} \frac{\partial \chi}{\partial v}\right)\right] \\
C_{i, e}(\chi)=-B(i, e)\left(\frac{2}{v_{t e}}\right)^{3} \frac{1}{m_{e}} \frac{\partial}{\partial v} \cdot\left[\frac{T}{M_{i}} f_{M} \frac{\partial \chi}{\partial v_{i}}\right]
\end{array}\right)
$$

where $\mathrm{L}$ is the Lorenz collision operator, $f_{1}=f_{M} \chi, v_{t e}$ is the electron thermal velocity, and $B(i, e)=(2 \sqrt{2} / 3 \sqrt{\pi}) A(i, e)$. From Eq.(10), if $\left(m_{e} / M_{i}\right)^{1 / 2}\left(E_{G} / T\right)^{1 / 6} \ll 1$, then $C_{i, e}$ can be ignored compared with $C_{i, i}$ for the particle with $E=E_{G p}$. The derivation and further explanation can be found in Appendix A. For several species of ions, simply ignoring ion-electron collisions, we obtain

$$
-\Sigma_{s} C_{i, s}\left(\chi_{i}\right)=\Sigma_{s} A(i, s)\left[\frac{f_{M}}{m_{i} v^{3}}\left(L^{2} \chi-2 v \frac{m_{i}}{m_{s}} \frac{\partial \chi}{\partial v}\right)\right]
$$

\section{THE REACTION RATE WHEN CEP IS VALID}

For simplicity, we consider an one-component plasma in this section; the treatment can be easily generalized to several components. We now solve Eq.(7) using Eqs.(8) and (10). Two sources in Eq.(8) are the temperature gradient and the velocity shear, $\chi_{h}$ driven by the temperature gradient, and $\chi_{s}$ by the velocity shear, so that

$$
\left(\begin{array}{c}
\chi_{h}(\mathbf{v})=\tau(v)\left[\frac{-1}{16} \frac{v^{2}}{v_{t}^{2}}+\frac{5}{8}\right] v \cdot \nabla \ln T \\
\chi_{s}(\mathbf{v})=\tau(v)\left(\frac{-1}{10}\right) \Sigma_{i, j}\left(\frac{v_{i} v_{j}}{v_{t}{ }^{2}}-\frac{1}{3} \frac{v^{2}}{v_{t}^{2}} \delta_{i, j}\right): \hat{S}_{i, j}
\end{array}\right)
$$


where $v_{t}=\sqrt{T_{i} / m}$ is the thermal velocity, and $\tau(v)=\frac{1}{2 \pi}\left(m / n e^{4}\right)(1 / \ln \Lambda) m v^{3}$ is the collision time of the particle with the velocity $v$. Note that $\chi$ increases with energy, and exceeds 1 as $v$ gets large. As mentioned, this is a common limitation of the CEP.

In the case when the CEP is applicable, we can substitute $\chi$ into Eq.(2). By changing the variable from $v_{1}$ and $v_{2}$ into $V=\left(v_{1}+v_{2}\right) / 2$ and $v=v_{1}-v_{2}$, we obtain from Eq.(2)

$$
P=n^{2} \int\left[f_{M}(V) f_{\mu}(v)\left(1+\chi_{1}\right)\left(1+\chi_{2}\right) \sigma(v) v\right] d^{3} v d^{3} V
$$

where $f_{\mu}$ is a Maxwellian distribution with mass $\mu$.

For convenience, let us define $\langle a(v)\rangle_{g}=\int g(v) a(v) d^{3} v$, where $g(v)$ is any spherically symmetric function. We then note that $\langle a(v)\rangle_{g}=0$ if $a(-v)=-a(v),\left\langle v_{x} v_{y}\right\rangle_{g}=0$, and $\left\langle 2\left(v_{x}\right)^{2}-\left(v_{y}\right)^{2}-\left(v_{z}\right)^{2}\right\rangle_{g}=0$. Because $\chi_{h}$ is an odd function, and $\chi_{s}$ has the form $\chi(v)=$ $g(v) v_{x} v_{y}$ or $\chi(v)=g(v)\left(2 v_{x}^{2}-v_{y}^{2}-v_{z}^{2}\right)$, the first order correction to the rate in $\chi$ is zero.

The second order correction is not zero. To simplify the computation, we now make use of the fact that most of the reactions occur when $|v| \gg|V|$, because the center-of-mass energy is near $T$ and the relative energy is near the Gamow Peak. Thus approximations $V \pm v / 2 \cong \pm v / 2$ and $\chi(V \pm 2 / v) \cong \chi( \pm v / 2)$ can be used. The second order modification is then

$$
\frac{\delta P}{P_{0}}=-|\nabla \ln T|^{2} A+\hat{S}_{O}: \hat{S}_{O} B+\left(x^{2}+y^{2}+z^{2}-1 / 2(x z+y z+x y)\right) B^{\prime}
$$

where $\tau=\frac{1}{2 \pi}\left(m / n e^{4}\right)(1 / \ln \Lambda) m\left(v_{G p} / 2\right)^{3}$ is the collision time for a particle with the Gamow peak energy ( or the velocity $v_{G p}=\sqrt{E_{G p} / m}$ ), and $A=\tau^{2}(5 / 8)^{2}\left(E_{G p} / 12 m\right.$ ), $B=\tau^{2}\left(1 / 10^{2}\right)(1 / 15)\left(E_{G p} / 4 T\right)^{2}, B^{\prime}=4 / 3 B, x=\frac{\partial u_{x}}{\partial x}, y=\frac{\partial u_{y}}{\partial y}, z=\frac{\partial u_{z}}{\partial z}, \hat{S}_{O}(i, j)=$ $\hat{S}(i, j)-\hat{S}(i, j) \delta_{i, j}$ is the off-diagonal element of the velocity shear matrix, and $P_{0}(n, T)$ is given in Eq.(5). We assumed $\tau_{i, j}<10$ with $\tau_{i, j}$ in Sec. II.

We note from Eq.(14) that the rate modification mostly comes from the temperature gradient. If the shear effect is as big as the temperature-gradient effect, the macroscopic velocity would have to be as large as the Gamow peak velocity, which is unlikely. We note also that, in comparison with the local fusion $P_{0}$, the temperature gradient reduces the fusion rate and the velocity shear increases the rate. Most fusion reactions occur when two fusing particles have $V \cong 0$ and a large relative energy $v \cong \sqrt{2 E_{G p} / \mu}$; In this case, they 


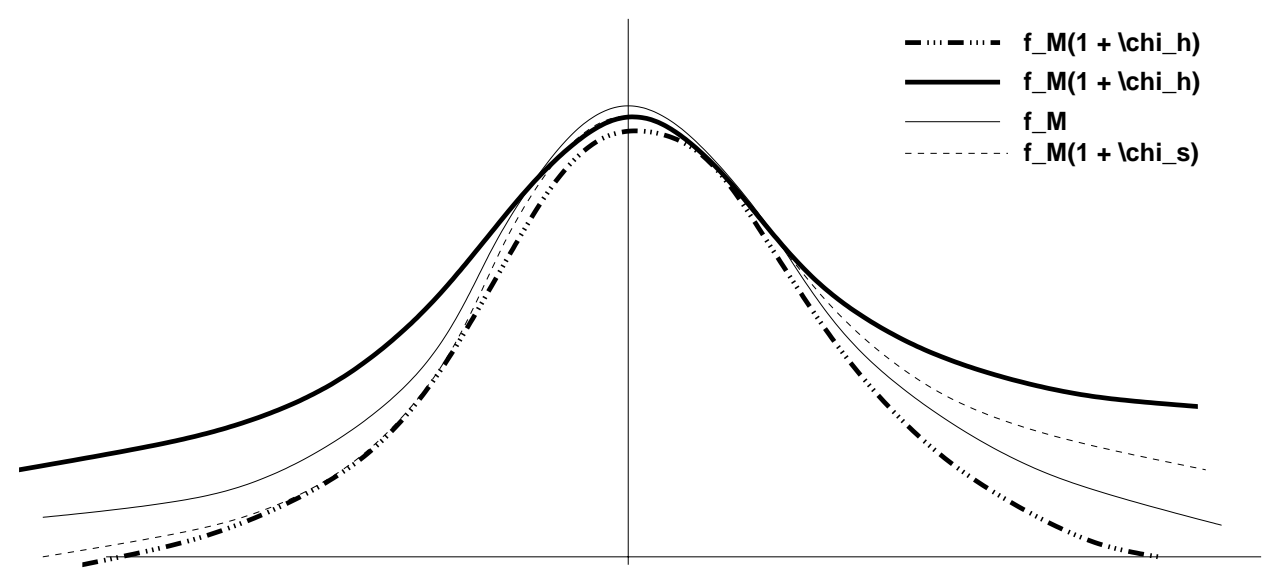

FIG. 1: $f_{M}, f_{M}\left(1+\chi_{s}\right), f_{M}\left(1+\chi_{h}\right)$.

fuse with nearly opposite velocities $v_{1} \cong-v_{2}$. Note that $\chi_{h}(v) \chi_{h}(-v)=-\left(\chi_{h}(v)\right)^{2}<0$ and $\chi_{s}(v) \chi_{s}(-v)=\left(\chi_{s}(v)\right)^{2}>0$. Since $\chi_{h}$ is an odd function of $v$, whenever $\chi_{h}$ has an abundance of density in a particular region of phase space, there will be a dearth of density in the corresponding opposite region $\left(-v_{x},-v_{y},-v_{z}\right)$. Since the reaction rate is proportional to the product of oppositely traveling particles, $\chi_{h}$ always offsets each other to make the reaction rate reduced. A similar argument can be applied to $\chi_{s}$ with the opposite effect. See Fig.(1).

Most of the fusion reactions occurs with the particles in the region near the Gamow Peak. CEP breaks down if $\chi$ is larger than or comparable to 1 in this region, rendering Eq.(14) invalid, Although this equation has then limited applicability, it serves as a check whether a local Maxwellian or CEP can be used. For example, consider a plasma with physical parameters $Z=1, n=10^{14} \mathrm{~cm}^{-3}, m_{1}=m_{2}=O(1), T=50 \mathrm{eV}$, and $E_{G p}=500 \mathrm{eV}$. For this plasma, $\tau=0.2 \times 10^{-3}$, and CEP is valid only when $\nabla \ln T<0.01 \mathrm{~cm}^{-1}$.

\section{SHOCK REGION WHEN CEP BREAKS DOWN}

Consider the shock region in Fig.(2). In Region $2(|x|<L)$, the density and the temperature change from $n_{0}, T_{0}$ to $n_{1}, T_{1}$ while maintaining the Euler equilibrium, $n T=$ Const. Of interest here is when $\lambda(T)<L<\lambda\left(E_{G p}\right)$. The distribution function in Region 2 can not then be obtained from CEP perturbation, but must be obtained instead from the solution to 


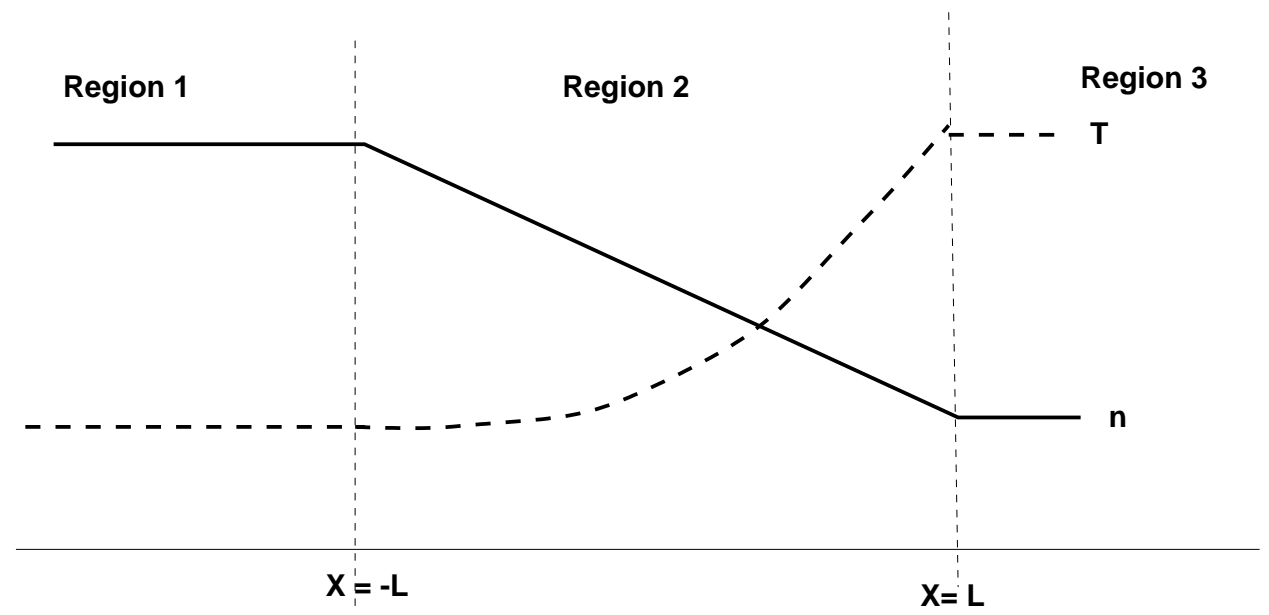

FIG. 2: The shock region.

$$
v_{x} \frac{\partial f_{E}}{\partial x}=-C\left(x, f_{E}\right)
$$

where $C(x)$ depends on $x$ because of $n(x)$ and $T(x)$. We estimate the rate in Region 2 without solving Eq.(15) by the following argument. If $L \ll \lambda\left(E_{G p}\right)$, energetic particles from Region 1 cannot be relaxed quickly enough while traversing Region 2, and so it still has the memory of the temperature and the density in Region 1. Particles from Region 3 act similarly in Region 2. Therefore, in Region 2, the particle distribution function $f_{2}$ can be considered as a Maxwellian with temperature $T_{0}$ when $v_{x}>0$ and a Maxwellian with $T_{1}$ when $v_{x}<0$ :

$$
f_{2}(\mathbf{v})=\left(\begin{array}{c}
f_{1}(\mathbf{v})\left(v_{x}>0\right) \\
f_{3}(\mathbf{v})\left(v_{x}<0\right)
\end{array}\right)
$$

where $f_{1}$ and $f_{3}$ are the particle distribution functions in Region 1 and Region 3. We can then put $f_{2}$ in Eq.(16) into Eq.(2), The rate between $g$ and $f$ particles in Region 2 is then

$$
R \cong \frac{1}{2} \int d v_{1}^{3} d v_{2}^{3} \sigma\left(\left|v_{1}-v_{2}\right|\right)\left|v_{1}-v_{2}\right|\left[f_{T_{0}}\left(v_{1}\right) g_{T_{1}}\left(v_{2}\right)+f_{T_{1}}\left(v_{1}\right) g_{T_{0}}\left(v_{2}\right)\right]
$$


where $f_{T_{0}}, g_{T_{0}}$ and $f_{T_{1}}, g_{T_{1}}$ are Maxwellian with temperatures $T_{0}, T_{1}$. After integrating over the center-of-mass coordinate, the rate is the same as the rate given in Eq.(5) with the effective temperature and the effective density given as

$$
\left(\begin{array}{c}
\frac{1}{T_{E 1}}=\frac{m_{1}+m_{2}}{\left(\frac{m_{1}}{T_{0}}+\frac{m_{2}}{T_{1}}\right)} \frac{1}{T_{1} T_{0}} \\
\frac{1}{T_{E 2}}=\frac{m_{1}+m_{2}}{\left(\frac{m_{2}}{T_{0}}+\frac{m_{1}}{T_{1}}\right)} \frac{1}{T_{1} T_{0}} \\
n_{E}=\sqrt{\frac{n_{0} n_{1}}{2}}
\end{array}\right)
$$

The total rate is then

$$
P_{T}=P\left(T_{E 1}, n_{E}\right)+P\left(T_{E 2}, n_{E}\right)
$$

where $P\left(T_{E 1}, n_{E}\right)$ is the reaction rate between particles $f_{1}$ from Region 1 and particles $g_{3}$ from Region 3 , and $P\left(T_{E 2}, n_{E}\right)$ is the reaction rate between particles $f_{3}$ from Region 3 and particles $g_{1}$ from Region 1. $P_{T}\left(T_{E}, n_{E}\right)$ is quite different from the local fusion power $P_{0}(T(x), n(x))$ in Eq.(5). We now check how $P_{0}$ differs from the true rate $P_{T}$ in Region 2. We note that when $n T=$ Const, $P_{0}$ in Eq.(5), as a function of $T$, has a maxima at $\tau_{i, j}=8$, at which $T_{m}=0.13 E_{G}$ from

$$
\frac{d P}{d T}=\frac{1}{T}\left(\frac{1}{3} \tau_{i j}-\frac{8}{3}\right) P
$$

For D-T plasma, $T_{m} \cong 15 \mathrm{KeV}$. For convenience, we identify three cases: $T_{0}<$ $T_{m}<T_{1}, T_{m}<T_{0}<T_{1}, T_{0}<T_{1}<T_{m}$. Instead of $\mathrm{T}$, a dimensionless parameter $a=(8 / 3)^{3}(2 / \pi)^{2}\left(T / E_{G}\right)$ will be used for simplicity. Note $a_{m}=1$ at $T_{m}$.

We consider reactions involving equal-mass ions such as D-D when $a_{0}<1<a_{1}$. In Fig.(3), $P / P_{0}$ is drawn in Region 2 when $a_{0}=0.5, a_{1}=1.3$. The actual fusion power is larger than $P_{0}$ in the entire region. This comes from the fact that $T_{E}=\left(T_{0}+T_{1}\right) / 2$, $\sqrt{2} n_{E} T_{E}=n_{0} T_{0}\left(T_{0}+T_{1}\right) / 2 \sqrt{T_{0} T_{1}}>n_{0} T_{0} . P_{0}$ underestimates the actual fusion power.

We now consider the case when there is a big mass difference between the fusing particles, such as P-Boron-11. Since $\left(m_{1} / T_{0}\right)>>\left(m_{2} / T_{1}\right)$ or $\left(m_{1} / T_{0}\right)<<\left(m_{2} / T_{1}\right)$, it is true that 


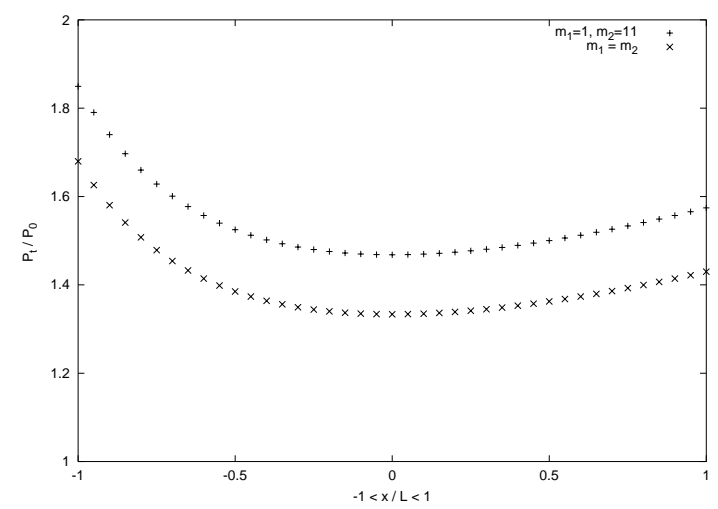

FIG. 3: $\frac{P_{t}}{P_{0}}$ in region 2 for $m_{1}=m_{2}$ and $m_{1}=1, m_{2}=11$ when $a_{0}=0.5, a_{1}=1.5$.

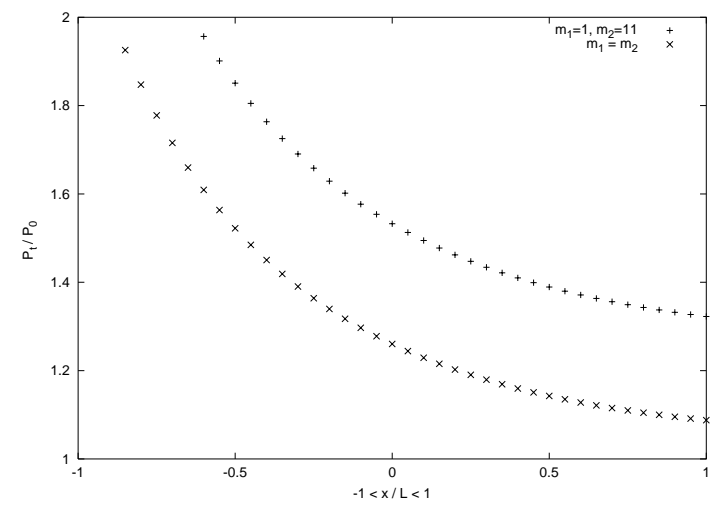

FIG. 4: $\frac{P_{t}}{P_{0}}$ in region 2 for $m_{1}=m_{2}$ and $m_{1}=1, m_{2}=11$ when $a_{0}=0.3, a_{1}=0.8$.

$T_{E 1} \cong T_{0}, T_{E 2} \cong T_{1}$. Then, $P_{T}=\frac{1}{2}\left[\left(T_{0} / T_{1}\right) P\left(T_{0}\right)+\left(T_{1} / T_{0}\right) P\left(T_{1}\right)\right]$. See Fig.(3) for a case in which $P_{0}$ underestimates the true rate in the entire region.

We also consider a case when $a_{0}<a_{1}<a_{m}$. The local fusion power usually underestimates the actual rate in the region near $x / L=-1$ and overestimates near $x / L=1$. However, it is possible that the local fusion power can underestimate the rate in entire Region 2 as shown in Fig.(4).

The same analysis can be applied to the case where $a_{m}<a_{0}<a_{1}$, but $\lambda(T)<L<\lambda\left(E_{G p}\right)$ is not easy to satisfy. Except for very limited cases, $\lambda(T)<\lambda\left(E_{G p}\right)<L$ or $L<\lambda(T)<$ $\lambda\left(E_{G_{p}}\right)$, so the estimation method in this section cannot be applied.

\section{DISCUSSION}

In a DT inertial confinement fusion, a detonation front [20-22] is a supersonic shock front. The following is a rough comparison of the width of the shock front with the mean 
free path of an ion at Gamow-Peak energy thereby to determine whether the non-locality is important. In a detonation scenario of DT fuel [22], an electron wave with $T_{e}=8 \mathrm{KeV}$ heats ions to the ignition temperature $T_{i}=5 \mathrm{KeV}$ [21]. Roughly, the mean free path of a $3.5 \mathrm{MeV}$ alpha particle and that of a deuterium with the energy $E$ is given as

$$
\left(\begin{array}{c}
\lambda_{\alpha}=0.1462 \times 10^{18} \frac{T_{e}^{1.5}}{n_{e} \lambda} \\
\lambda(E)=0.67 \times 10^{13} \frac{E^{2}}{n_{e} \lambda}
\end{array}\right),
$$

where $T$ and $E$ in $\mathrm{eV}$, and $n_{e}$ in $\mathrm{cm}^{-3}$. Then $\lambda(E) / \lambda_{\alpha}$ is

$$
\frac{\lambda(E)}{\lambda_{\alpha}}=4.56 \times 10^{-5} \frac{E^{2}}{T_{e}^{1.5}} .
$$

Assuming $T_{i} \cong 5 \mathrm{KeV}$, we note that $E_{G p}=28.4 \mathrm{KeV}$ and $\lambda\left(E_{G p}\right) / \lambda_{\alpha} \cong 0.1$. The width of the shock front is the same order as the mean free path of the Gamow-Peak ions $L\left(E_{G_{p}}\right)$, because it is much smaller than $\lambda_{\alpha}$. The non-local effect in Sec. IV is then important. However, for the internal structure of the shock, we used the simple static isobaric configuration. A more refined study [7, 23] should be carried out especially for the fast igniter scheme [22, 24-26].

In P-Boron-11 [21], the ion temperature $T_{i} \gtrsim 100 \mathrm{KeV}$ must be much hotter than the electron temperature $T_{e} \cong 30 \mathrm{KeV}$ to overcome the bremsstrahlung losses [27]. The operating regime of a detonation waves in P-B-11 fuel is quite narrow [21]. The plausibility of the existence of this region is called into question due to the reduced reactivity compared with the old data [28]. However, as shown in this work, the non-locality of fusing particles can enhance the fusion power as much as $100 \%$ in some region. We note that $\lambda\left(E_{G p}\right)$ is not much smaller than $\lambda_{\alpha}$ and might be larger than the width of the shock front because the ion temperature is above $100 \mathrm{KeV}$. Therefore, by having a sharp front, the fusion rate might be higher than the theoretical prediction, and a detonation wave might be possible. It is then important to study P-B-11 with inclusion of the non-locality effect.

For the case of the nuclear reaction rate, the non-locality effect has been generally ignored. The reason is that the nuclear reaction rate is usually slow compared to the other physical processes involved in astrophysical plasmas [29] and laboratory fusion devices. Even a sudden change of the plasma parameters for a short period of time can be simply ignored. But with 
the increasing interest in ICF, this very short period becomes quite important, especially in the shock front, because the possibility that the detonation depends on the characteristic of the shock. A thorough analytical formulation has not apparently been carried out yet. Some treatment has been made to take into account the non-locality from Chapman-Enskog procedure or other method [7, 23], whose primary interest is particle flux or current. We need however the detailed information about the distribution at Gamow peak, and no formalism has been developed for this purpose to our knowledge.

\section{CONCLUSION}

In this paper, the modification of the fusion reaction rate due to plasma inhomogeneities was calculated. If an energetic particle with the Gamow-Peak velocity travels a sufficient distance to see the spatial inhomogeneity before being collisionally damped, the reaction rate formula $P_{0}(n, T)$ should be changed considerably.

The modification $\delta P(n, \nabla n, T, \nabla T, V, \nabla V)$ has been calculated using the Chapman Enskog procedure when the inhomogeneity is small. In the presence of a temperature gradient, the local fusion rate overestimates the actual fusion rate. In the presence of a velocity shear, the local fusion rate underestimates the actual rate. CEP also serves as a criteria to determine when the perturbation procedure breaks down.

The case when $\delta P / P_{0} \cong O(1)$ is given in the shock region, and a qualitative description has been made. The modification to fusion rates can be large, and the local fusion power formula can underestimate or overestimate the actual rate.

We would like to thank Dr. John Krommes for useful discussion. This work was supported by the U.S. DOE under contract AC02-76CH0-3073.

\section{Appendix [5]}

\section{A. Electron-Ion Collisions and Ion-Ion Collisions}

We compare the magnitude between $C_{i, e}$ and $C_{i, i}$ when $E_{i} \cong E_{G p}$ in order to check whether the linearization doesn't omit the important terms. From $v_{G p}=\sqrt{\frac{1}{3} \tau_{i j}(T / \mu)} \ll v_{t e}$, where $v_{t e}$ is the thermal velocity of the electron, the ratio of the drag on an ion by the electrons to the drag by the ions is 


$$
\frac{\nu^{i, e}}{\nu^{i, i}}=1.78 \times 10^{-2} m^{-1 / 2}\left(\frac{E}{T}\right)^{3 / 2}
$$

where $m$ is the ion mass in esu, and $\mathrm{E}$ is the energy of the ion. According to Eq.(23), $C_{i, e}$ can be ignored for the energetic ion if $E<T \times\left(10^{2} / 1.78\right)^{2 / 3}$. We impose this condition to the particle with $E=E_{G p}$, and we obtain $E_{G} / T<1200$. For D-T plasma, it happens when the temperature is hotter than $100 \mathrm{eV}$. As shown in the linearized version, we can ignore ion-electron collisions as long as $T \gg E_{G} / 2000^{3}$. This is a much stronger condition than $T>100 \mathrm{eV}$. We indeed omitted some important physics when we linearized it around a Maxwellian, but not seriously. The above analysis is consistent with an ordering between collision operators:

$$
C_{e, e}, C_{e, i}: C_{i, i}: C_{i, e} \cong 1:\left(\frac{m}{M}\right)^{1 / 2}: \frac{m}{M} .
$$

Therefore, we can ignore $C_{i, e}$ compared to $C_{i, i}$.

\section{B. Ion-Electron Collision Operator $C_{i, e}$}

If thermal electrons are much faster than the ions so that $v_{i} \ll v_{t h e}, \hat{U}$ can be approximated as $\hat{U}_{k, p}\left(\overrightarrow{v_{e}}-\overrightarrow{v_{i}}\right) \cong \hat{U}_{k, p}\left(v_{e}\right)-\Sigma_{q}\left(v_{i}\right)_{q} \cdot \frac{\partial \hat{U}_{k, p}\left(v_{e}\right)}{\partial v_{q}}$. Using this, $C_{i, e}$ becomes

$$
\begin{aligned}
C_{i, e} \cong-A_{i, e} \Sigma_{k, p}\left(\frac{\partial}{\partial v}\right)_{k} \cdot \int & {\left[\hat{U}_{k, p}\left(v_{e}\right)-\Sigma_{q}\left(v_{i}\right)_{q} \cdot\left(\frac{\partial}{\partial v}\right)_{q} \hat{U}_{k, p}\left(v_{e}\right)\right] } \\
& {\left[\frac{1}{m_{i}}\left(\frac{\partial f_{i}}{\partial v}\right)_{p} f_{e}-\frac{1}{m_{e}}\left(\frac{\partial f_{e}}{\partial v}\right)_{p} f_{i}\right] d^{3} v_{e} }
\end{aligned}
$$

Keeping only the largest term in order $O\left(m_{e} / m_{i}\right)$, the expression becomes

$$
\begin{gathered}
C_{i, e}\left(f_{i}(v)\right)=-A_{i, e} \Sigma_{k}\left(\frac{\partial}{\partial v}\right)_{k} \cdot\left[\vec{M}_{k}+\vec{N}_{k}+\vec{L}_{k}\right] \\
\left(\begin{array}{c}
\vec{M}_{k}=\Sigma_{p} \frac{1}{T_{e}} f_{i}\left\langle\hat{U}_{k, p}\left(v_{e}\right) \cdot\left(v_{e}\right)_{p}\right\rangle \\
\vec{N}_{k}=-\Sigma_{p, q} \frac{1}{T_{e}} f_{i} v_{q} \cdot\left\langle\left(\left(\frac{\partial}{\partial v_{e}}\right)_{q} \hat{U}_{k, p}\left(v_{e}\right)\right\rangle\right. \\
\vec{L}_{k}=\Sigma_{p} \frac{1}{m_{i}}\left(\frac{\partial f_{i}}{\partial v}\right)_{p} \cdot\left\langle\hat{U}_{k, p}\left(v_{e}\right)\right\rangle
\end{array}\right),
\end{gathered}
$$


where \langle\rangle denote the Maxwellian integration with respect to the electron velocity. Since $\left\langle\frac{\partial \hat{U}_{k, p}\left(v_{e}\right)}{\partial v_{e}} \cdot v_{e}\right\rangle=a \delta_{k, p},\left\langle\hat{U}_{k, p}\right\rangle=-a \delta_{k, p}$, and $\left\langle\hat{U}_{k, p}\left(v_{e}\right) \cdot v_{e}\right\rangle=0$ where $a=-\frac{8 \pi}{3}(2 \pi)^{-3 / 2} \sqrt{m_{e} / T_{e}}$, $C_{i, e}$ is finally given

$$
C_{i, e}\left(f_{i}\right)=-B_{i, e}\left(\frac{2}{v_{t e}}\right)^{3} \frac{1}{m_{e}} \frac{\partial}{\partial v} \cdot\left[v_{i} f_{i}+\frac{T}{M_{i}} \frac{\partial\left(f_{i}\right)}{\partial v_{i}}\right]
$$

where $B_{i, e}=(2 \sqrt{2} / 3 \sqrt{\pi}) A_{i, e}$.

\section{Ion-Ion Collision Operator $C_{i, j}\left(\chi^{i}\right)$}

By using $f^{i}=\left(1+\chi^{i}\right) f_{m}^{i}$, where $f_{m}^{i}$ is a Maxwellian of the species $i$, we obtain the linearized Landau operator

$$
C_{i, j}\left(\chi^{i}\right)=-A_{i, j} \Sigma_{k, p}\left(\frac{\partial}{\partial v}\right)_{k} \cdot\left[f_{m}^{i}(v) \int\left[f_{m}^{j}(\bar{v}) \hat{U}_{k, p}(v-\bar{v}) \cdot\left(\frac{1}{m_{i}} \frac{\partial \chi^{i}}{\partial v_{p}}-\frac{1}{m_{j}} \frac{\partial \chi^{j}}{\partial \bar{v}_{p}}\right)\right] d^{3} \bar{v}\right]
$$

The integral $\hat{H}(v)=\int f_{m}^{j}(\bar{v}) \hat{U}_{k, p}(v-\bar{v})$ can be set as $\hat{H}_{k, p}=a^{j}(v)\left(\delta_{k, p}-\vec{v}_{k} \vec{v}_{p}\right)+b^{j}(v) \vec{v}_{k} \vec{v}_{p}$ where $b^{j}(v)$ and $a^{j}(v)$ can be obtained from

$$
\left(\begin{array}{c}
a^{j}(v)=\frac{1}{2}\left[\Sigma_{k, p} \hat{H}_{k, p}-b(v)\right] \\
b^{j}(v)=\Sigma_{k, p} \vec{v}_{k} \cdot \hat{H}_{k, p} \cdot \vec{v}_{p} \\
\Sigma_{k, p} \hat{H}_{k, p}=\frac{2}{v} g\left(\frac{v}{\sqrt{2} v_{t}}\right)
\end{array}\right),
$$

where $g(x)=\frac{2}{\pi} \int_{0}^{x} e^{-t^{2}} d t$. From this, $a(v)$ and $b(v)$ is

$$
\left(\begin{array}{c}
a(x)=\frac{1}{v_{t j}} \frac{1}{x^{3}}\left[\left(x^{2}-1\right) g\left(\frac{x}{\sqrt{2}}\right)+\sqrt{\frac{2}{\pi}} x e^{-\frac{1}{2} x^{2}}\right] \\
b(x)=\frac{1}{v_{t j}} \frac{2}{x^{3}}\left[g\left(\frac{x}{\sqrt{2}}\right)-\sqrt{\frac{2}{\pi}} x e^{-\frac{1}{2} x^{2}}\right]
\end{array}\right)
$$

where $x=v / v_{t j}$, and $v_{t j}=\sqrt{T / m_{j}}$. After substituting Eqs.(29),(28) into Eq.(27), then Eq.(27) becomes 


$$
C_{i, j}\left(\chi_{i}(v)\right)=-A_{i, j} \Sigma_{k, p} \frac{\partial}{\partial v_{k}} \cdot\left[f_{m}\left(\hat{H}_{k, p} \cdot \frac{1}{m_{i}} \frac{\partial \chi}{\partial v_{p}}-\vec{J}_{k}(v)\right)\right],
$$

where $\vec{J}_{k}=\Sigma_{p} \int f_{m}(\bar{v}) \hat{U}_{k, p}(v-\bar{v}) \cdot\left(\frac{1}{m} \frac{\partial \bar{\chi}}{\partial \bar{v}_{p}}\right) d^{3} \bar{v}$. For $x \gg 1, b^{j}$ can be ignored compared to $a^{j}$. The last term is for momentum conservation. Since high energy particles can be considered as test particles due to their small number and the momentum conservation is not important, this term can be ignored. Mathematically, if one solves CEP without the last term and substitutes the solution into it, the last term is shown to be small compared to $\left[f_{m} v \cdot \ln T\right]$ and other terms. We then obtain the final expression

$$
C_{i, j}\left(\chi_{i}(v)\right)=-A_{i, j} \Sigma_{k, p} \frac{\partial}{\partial v_{k}} \cdot\left[f_{m} \cdot \hat{H}_{k, p} \cdot \frac{1}{m_{i}} \frac{\partial \chi}{\partial v_{p}}\right],
$$

where $\hat{H_{k, p}}=\frac{1}{v}\left(\delta_{k, p}-\vec{v}_{k} \vec{v}_{p}\right)$. After linearizing Eq.(31), we obtain Eq.(10).

[1] S.I. Braginskii, Review of Plasma physics, Volume 1, 205 ( Consultant Bureau, New York, $1965)$.

[2] P.V. Sivukhin, Review of Plasma physics, Volume 1, 1 ( Consultant Bureau, New York, 1965 ) .

[3] B.A. Trubinikov, Review of Plasma physics, Volume 1, 105 ( Consultant Bureau, New York, 1965).

[4] S. Chapman and T.G. Cowling, The mathematical theory of non-uniform gases, ( Cambridge University Press, 1956 ).

[5] J.A. Krommes, Lecture note in irreversible processes in plasma, ( In preparation, 2000).

[6] L. Spitzer, Jr. and R. Harm, Physical Review 89, 977 ( 1953 ).

[7] N.J. Fisch, Phys. Fluids. 29, 172 ( 1985 ).

[8] J.P. Matte, T.W. Johnston, J. Delettrez, and R.L. McCrory, Rhysical Review Letters 53, 1461 ( 1984 ).

[9] F. Vidal, J.P. Matte, M. Casanova and O. Larroche, Physical Review E 52, 4568 ( 1995 ).

[10] M. Casanova, O. Larroche and J.P. Matte, Physical Review Letters 67, 2143 ( 1991 ).

[11] A.V. Brantov and V. Yu. Bychenkov, Phys. Plasmas 10, 4633 ( 2003 ). 
[12] E. Fourkal and V.Yu. Bychenkov, Phys. Plasmas 8, 550 ( 2001 ).

[13] O.V. Batishchev and V.Yu. Bychenkov, Phys. Plasma 9, 2302 ( 2002 ).

[14] A.R. Bell, Phys. Fluid. 26, 279 ( 1983 )

[15] A. B. Langdon, Physical Review Letters 44, 575 ( 1980 ).

[16] E.M. Epperlein and R.W. Short, Phys. Fluid. B. 4, 2211 ( 1992 ).

[17] E.M. Epperlein and R.W. Short, Physical Review E 50, 1697 ( 1994 ).

[18] S. Ichimaru, Rev. Mod. Phys. 65, 255 ( 1993 ).

[19] G. Gamow and E. Teller, Phys. Rev. 53, 608 ( 1938 ).

[20] J.M. Martinez-Val and S. Eliezer and M. Piera, Laser and Particle Beams 12, 681 (1994).

[21] J.M. Martinez-Val and S. Eliezer and M. Piera and G. Velarde, Physics Letters A 216, 142 (1996).

[22] M. S. Chu, Phys. Fluids. 15, 413 ( 1972 ).

[23] R.D. Hazeltine, Phys. Plasmas. 5, 3282 ( 1998 ).

[24] R.E. Kidder, Nuclear Fusion 15, 405 ( 1976 ).

[25] K.A. Brueckner and S. Jorna, Rev. Mod. Phys. 46, 325 ( 1974 ).

[26] M. Tabak, Phys. Plasmas 1, 1626 ( 1994 ).

[27] J. Dawson, Fusion, Volume 2, ( Academic Press, New York, 1981 ).

[28] W.M. Nevins and R. Swain, Nuclear Fusion 40, 865 ( 2000 ).

[29] R.Rosner, Com. Sci. Eng. 2, 33 ( 2000 ). 


\section{External Distribution}

Plasma Research Laboratory, Australian National University, Australia

Professor I.R. Jones, Flinders University, Australia

Professor João Canalle, Instituto de Fisica DEQ/IF - UERJ, Brazil

Mr. Gerson O. Ludwig, Instituto Nacional de Pesquisas, Brazil

Dr. P.H. Sakanaka, Instituto Fisica, Brazil

The Librarian, Culham Laboratory, England

Mrs. S.A. Hutchinson, JET Library, England

Professor M.N. Bussac, Ecole Polytechnique, France

Librarian, Max-Planck-Institut für Plasmaphysik, Germany

Jolan Moldvai, Reports Library, Hungarian Academy of Sciences, Central Research Institute for Physics, Hungary

Dr. P. Kaw, Institute for Plasma Research, India

Ms. P.J. Pathak, Librarian, Institute for Plasma Research, India

Ms. Clelia De Palo, Associazione EURATOM-ENEA, Italy

Dr. G. Grosso, Instituto di Fisica del Plasma, Italy

Librarian, Naka Fusion Research Establishment, JAERI, Japan

Library, Laboratory for Complex Energy Processes, Institute for Advanced Study, Kyoto University, Japan

Research Information Center, National Institute for Fusion Science, Japan

Dr. O. Mitarai, Kyushu Tokai University, Japan

Dr. Jiangang Li, Institute of Plasma Physics, Chinese Academy of Sciences, People's Republic of China

Professor Yuping Huo, School of Physical Science and Technology, People's Republic of China

Library, Academia Sinica, Institute of Plasma Physics, People's Republic of China

Librarian, Institute of Physics, Chinese Academy of Sciences, People's Republic of China

Dr. S. Mirnov, TRINITI, Troitsk, Russian Federation, Russia

Dr. V.S. Strelkov, Kurchatov Institute, Russian Federation, Russia

Professor Peter Lukac, Katedra Fyziky Plazmy MFF UK, Mlynska dolina F-2, Komenskeho Univerzita, SK-842 15 Bratislava, Slovakia

Dr. G.S. Lee, Korea Basic Science Institute, South Korea

Institute for Plasma Research, University of Maryland, USA

Librarian, Fusion Energy Division, Oak Ridge National Laboratory, USA

Librarian, Institute of Fusion Studies, University of Texas, USA

Librarian, Magnetic Fusion Program, Lawrence Livermore National Laboratory, USA

Library, General Atomics, USA

Plasma Physics Group, Fusion Energy Research Program, University of California at San Diego, USA

Plasma Physics Library, Columbia University, USA

Alkesh Punjabi, Center for Fusion Research and Training, Hampton University, USA

Dr. W.M. Stacey, Fusion Research Center, Georgia Institute of Technology, USA

Dr. John Willis, U.S. Department of Energy, Office of Fusion Energy Sciences, USA

Mr. Paul H. Wright, Indianapolis, Indiana, USA 
The Princeton Plasma Physics Laboratory is operated by Princeton University under contract with the U.S. Department of Energy.

\author{
Information Services \\ Princeton Plasma Physics Laboratory \\ P.O. Box 451 \\ Princeton, NJ 08543
}

Phone: 609-243-2750

Fax: 609-243-2751

e-mail: pppl_info@pppl.gov

Internet Address: http://www.pppl.gov 